\title{
Salmonella arteritis: an unusual cause of low back pain
}

D Pyne, R Mootoo, A Bhanji, A Farrow

Salmonella arteritis is a rare condition, first reported in $1948 .^{1}$ Salmonella typhimurium is responsible for most cases and here we report a case of $S$ typhimurium septicaemia with subsequent arteritis and aneurysm formation.

\section{Case report}

A 70 year old Afro-Caribbean man, previously fit and well, was admitted to hospital with a two month history of malaise, anorexia, weight loss of $6.5 \mathrm{~kg}$, constipation, low lumbar back pain, and frequency of micturition. The back pain was a dull, constant ache, present day and night, without radiation. He was not receiving regular drug treatment. He smoked 20 cigarettes a day.

On initial examination he was pyrexial at $38^{\circ} \mathrm{C}$, pulse rate 100 beats/min, normotensive, and dehydrated. Cardiovascular and respiratory examination were normal. There was suprapubic tenderness but no abdominal masses or organomegaly. The lower lumbar spine was tender and range of movement was limited in all directions as a result of pain. Investigations showed haemoglobin $102 \mathrm{~g} / \mathrm{l}$, mean cell volume $74 \mathrm{fl}$, white cell count $12.1 \times 10^{9} / 1$ (neutrophils $84 \%$ ), erythrocyte sedimentation rate $42 \mathrm{~mm} / 1 \mathrm{st} \mathrm{h}, \mathrm{C}$ reactive protein (CRP) $326 \mathrm{mg} / \mathrm{l}$, urine dipstix trace leucocytes and no proteinuria, and blood cultures showed no growth. A lumbar spine $x$ ray examination showed degenerative changes and erosion of the end plate between $\mathrm{L} 4$ and L5 vertebrae. A bone scan was normal. Culture from the urine grew $S$ typhimurium, and ciprofloxacin $500 \mathrm{mg}$ twice a day was started orally. Over the next week the patient improved, and ciprofloxacin was discontinued after a course of 10 days.

Three days later the haemoglobin fell suddenly to $78 \mathrm{~g} / \mathrm{l}$. There was no overt bleeding or cardiovascular compromise. The abdomen was non-tender with no masses, and a rectal examination was normal. The source of the bleed was unclear. Two units of blood were transfused. Oesophagogastro-duodenoscopy showed only mild gastritis, unlikely to explain the sudden anaemia, and a colonoscopy was normal.

The following week the patient became pyrexial again $\left(38^{\circ} \mathrm{C}\right)$. Repeat cultures of the urine were negative but blood cultures grew $S$ typhimurium. On re-examination of the patient

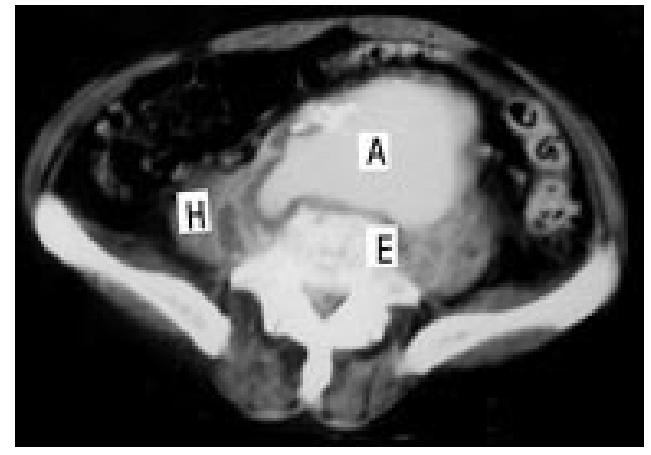

Figure 1 Computed tomography showing contrast-enhancing abdominal aneurysm $(A)$, haemorrhagic changes $(H)$ in the psoas, and erosive changes (E) in the body of L5 vertebrae.

there was now a pulsatile expansile mass in the left iliac fossa. Abdominal computed tomography (CT) showed a $6 \mathrm{~cm}$ common iliac artery aneurysm with haemorrhagic changes in the psoas consistent with a previous bleed. There was erosion of the body of L5 vertebra (fig 1). A mycotic aneurysm was suspected and ciprofloxacin orally was restarted together with gentamicin intravenously. The patient was transferred to a vascular unit, where repair of the aneurysm was undertaken. Surgery was complicated by aneurysm rupture, requiring massive blood transfusion and intensive care management. Histology from the aneurysm confirmed its mycotic nature with infiltration of its wall with neutrophils, although organisms were not isolated. Ciprofloxacin was continued for two weeks postoperatively. The patient improved and became apyrexial with a normal CRP. Subsequent rehabilitation for mobility was slow, and nine months after the initial illness he developed an overwhelming bronchopneumonia and died.

\section{Discussion}

Salmonella species are Gram negative bacilli. Infection, usually after ingestion of contaminated foodstuffs, can cause four groups of clinical manifestations ${ }^{2}$ : (a) gastroenteritis $(68 \%$ cases); (b) enteric fever $(9 \%) ;(c)$ septicaemia with or without focal extraintestinal infections (7\%); and (d) an asymptomatic carrier state $(15 \%)$. Septicaemia is often not preceded by gastrointestinal symptoms.

After invasion of the bloodstream Salmonella species can cause a variety of focal infections, ${ }^{2}$ 
including osteomyelitis, pyelonephritis, pneumonia, endocarditis, meningitis, and arteritis with or without subsequent aneurysm formation. $^{34}$

Infection of arteries may occur from a septic embolus as a complication of infective endocarditis, or extension from an adjacent suppurative process, ${ }^{5}$ or from lodgement of circulating organisms in the wall of an artery. The abdominal aorta and the femoral arteries are the most commonly affected, with very few cases arising above the renal arteries. ${ }^{6}$ Male sex and pre-existing atherosclerosis are risk factors for salmonella arteritis and the condition is extremely uncommon below the age of $50 .^{7}$ The onset of arteritis is usually insidious with gradual onset of low back pain and fever. Unlike atherosclerotic aneurysms, which tend to be asymptomatic unless they leak or rupture, the expansion of inflammatory abdominal aneurysms without leakage can cause a constant dull lumbar ache. Unlike true spinal pain, lumbar movement will not alter this pain. Abdominal examination may be normal initially, but progress can be rapid with the average time to aneurysmal rupture from onset of illness being five weeks. ${ }^{6}$ In our patient the initial normal abdominal examination lends support to the de novo formation of the aneurysm secondary to infection. Contributory causes of pain in this case, shown by CT, were bleeding into the psoas, which would have led to psoas spasm, and erosion of the L5 vertebral body. The latter was most probably secondary to concomitant salmonella infection of the bone.

Early diagnosis of salmonella arteritis, with ultrasound or CT scanning with contrast, is essential as $40 \%$ of patients die of vessel rupture or sepsis before surgery. ${ }^{4}$ Furthermore, arterial rupture can occur even before wall dilatation. $^{8}$
The mainstay of treatment is surgery. There have been no cases of long term survival using antibiotics alone. ${ }^{9}$ The procedure of choice is resection of the aneurysm with a vascular bypass through a non-septic tissue plane. ${ }^{10}$ This should be combined with the use of antibiotics for at least six weeks.

\section{Lessons}

- Arteritis can complicate salmonella septicaemia, the commonest causative agent being $S$ typhimurium.

- A high degree of awareness is necessary as the condition presents insidiously with fever and low back pain.

- Progress can be rapid with aneurysm formation and rupture.

- Management requires surgery and antibiotics.

1. Dehlinger KR. Salmonella osteomyelitis of the spine associated with abdominal aortic aneurysm: report of a case. N Engl J Med 1948;238:728-32.

2 Saphra I, Winter JW. Clinical manifestations of salmonellosis in man. N Engl J Med 1957;256:1128-34.

3 Winocour PH, Williams GR, Boyd JF, Kennedy DH. Septic arteritis complicating salmonellosis. BMJ 1983;287:972-3.

4 Lane GP, Cochrane AD, Fore DR. Salmonella mycotic abdominal-aortic aneurysm. Med J Aust 1988;149:95-7.

5 Reichle FA, Tyson RR, Soloff LA, Lautsch EV, Rosemond GP. Salmonellosis and aneurysm of the distal abdominal aorta. Ann Surg 1970;171:219-28.

6 Wilson SE, Gordon HE, Van Wegenen PB. Salmonella arteritis: a precursor of aortic rupture and pseudoaneurysm formation. Arch Surg 1978;113:1163-6.

7 Cohen PS, O'Brien TF, Schoenbaum SC, Medeiros AA. The risk of endothelial infection in adults with salmonella bacteraemia. Ann Intern Med 1978;89:931-2.

8 Cook AM, Christopoulos D. Rupture of a non-aneuysmal salmonella infected aorta. Clin Radiol 1989;40:605-6.

9 Ljungberg B, Braconier JH. Abdominal aortitis and infected aneurysms due to salmonella. Scand J Infect Dis 1986;18: 401-8.

10 Finseth F,Abbott WM. One stage operative therapy for salmonella mycotic abdominal aneurysm. Ann Surg 1974 179:8-11. 